\title{
Osteotomies in the Cervical Spine
}

\author{
Venu M. Nemani ${ }^{1}$, Peter B. Derman ${ }^{2}$, Han Jo Kim ${ }^{2}$ \\ ${ }^{1}$ Department of Orthopaedic Surgery, Washington University School of Medicine, Saint Louis, MO, USA \\ ${ }^{2}$ Spine Service, Hospital for Special Surgery, New York, NY USA
}

Rigid cervical deformities are difficult problems to treat. The goals of surgical treatment include deformity correction, achieving a rigid fusion, and performing a thorough neural decompression. In stiff and ankylosed cervical spines, osteotomies are required to restore sagittal and coronal balance. In this chapter, we describe the clinical and radiographic workup for patients with cervical deformities, and delineate the various factors that must be considered when planning surgical treatment. We also describe in detail the various types of cervical osteotomies, along with their surgical technique, advantages, and potential complications.

Keywords: Cervical spine; Deformity; Kyphosis; Myelopathy; Osteotomy

\section{Introduction}

Cervical deformities are among the most challenging problems that confront spinal surgeons and patients alike. They can cause significant functional disability and pain causing difficulty with forward gaze as well as dysphagia, both of which can have a significant impact on health related quality of life (HRQoL). In addition, they can also result in myelopathy and/or radiculopathy further disabling patients due to gait ataxia, manual dexterity issues and bowel/bladder dysfunction.

Cervical kyphosis is the most common deformity although scoliosis can also be present. While flexible deformities can be treated with a variety of surgical options, patients with rigid, ankylosed cervical spines require an osteotomy for appropriate deformity correction and neural decompression. The osteotomies that can be performed in the cervical spine are largely similar to those performed in the thoracolumbar spine; however, anatomic constraints (e.g., vertebral artery) make these more challenging to perform and with a higher risk of compli- cations. Here we describe a systematic approach for evaluating patients with cervical spine deformities and provide an algorithm to formulate a surgical treatment plan. In addition, we review the various types of cervical and our surgical technique for performing them safely.

\section{Clinical Symptoms \& Natural History}

The signs and symptoms of patients with symptomatic cervical deformity fall into three inter-related categories: (1) deformity, (2) neurologic, and (3) pain.

\section{Deformity}

Although multiplanar deformities can occur in the cervical spine, kyphosis is the most common may be attributable to one of several etiologies. These include degenerative spondylosis, iatrogenic (e.g., post-laminectomy), post-traumatic, infection, inflammatory arthritides (e.g., ankylosing spondylitis), neuromuscular diseases, and metabolic diseases [1-5]. Cervical kyphosis is typically

Received Jul 5, 2015; Accepted Jul 6, 2015

Corresponding author: Han Jo Kim

Spine Service, Hospital for Special Surgery, 535 E 70th St, New York, NY 10021, USA

Tel: +1-212-774-2837, Fax: +1-646-797-8428, E-mail: hanjokimmd@gmail.com 
progressive, and leads to abnormal positioning of the skull center of mass anterior to the occiput- $\mathrm{C} 1$ joints. As the deformity progresses and becomes more rigid, patients can develop pain, myelopathy, radiculopathy, difficulty achieving horizontal gaze or upright posture, swallowing dysfunction causing aspiration, social impairment, and even chest pressure sores in severe cases [4,5]. Pain may be attributable to degeneration of facet joints and intervertebral discs, compression of neural structures, as well as to increased stress on the posterior the soft tissue constraints (i.e., ligaments/muscles) as they struggle to maintain normal alignment $[4,5]$.

\section{Neurologic}

Although cervical deformity can occur in isolation, it is commonly accompanied by progressive myelopathy and/ or radiculopathy [6]. As the cervical spine develops progressively more kyphosis, the spinal cord is stretched and flattened against the posterior aspect of the vertebral bodies, leading to microvascular disruption, demyelination, and neuronal loss $[7,8]$. Additionally, cadaveric studies have shown significant increases in intramedullary pressure in the cervical spinal cord as the degree of kyphosis passes a critical threshold [9]. Compounding the increase in direct anterior pressure, there is also an increase in longitudinal cord tension due to the tethering of the cervical cord by the dentate ligaments and the cervical nerve roots $[6,10]$, which further increases intramedullary pressure leading to neuronal loss and demyelination [11-13]. Deformity patients thus frequently experience symptoms typical of myelopathy, such as gait instability, neck pain, diminished manual dexterity, bladder and bowel dysfunction, paresthesias and extremity weakness [6,14-18].

Studies of nonoperative management of cervical myelopathy reveal that myelopathy symptoms tend to progress without surgery [19]. While these studies were performed in patients suffering from myelopathy due to cervical spondylosis, not all of whom necessarily suffered from deformity, myelopathy secondary to cervical deformity likely has a similar natural history with a poor prognosis without operative intervention.

\section{Pain}

In addition to the deformity and neurologic symptoms, patients with cervical deformity also frequently suffer from neck pain. Progressive deformity leads to a shift of the weight-bearing axis of the head anterior to the cervical spine. This results in an increase in cantilever loads, necessitating increased cervical paraspinal muscular expenditure in an attempt to extend the neck to maintain horizontal gaze, which can cause muscular fatigue and resultant pain. Patients with cervical kyphosis frequently complain of neck and upper shoulder pain for these reasons. The semispinalis cervicis and capitis muscles, in particular, have been shown to be critically important in maintaining neutral head position [20]. In addition to neck pain, patients can also have lower back pain as they attempt to compensate for a rigid chin-on-chest deformity by bending their knees, increasing pelvic retroversion and increasing lumbar lordosis [21].

\section{Physical Exam}

The physical examination of the patient with cervical deformity should begin with a careful assessment of his or her global spinal alignment $[2,22,23]$. Normal alignment places the head centered over the pelvis with approximately $40^{\circ}$ of cervical lordosis, $50^{\circ}$ of thoracic kyphosis, and $60^{\circ}$ of lumbar lordosis in the sagittal plane, and no deviation from midline in the coronal plane [24]. The patient should be observed while standing upright, sitting, prone, and in supine and/or hyperextension positions. This allows the physician to identify any spinal deformities and coexistent hip/knee pathology, and to determine if these are rigid or flexible deformities [2,25].

Moving specifically to the examination of the cervical spine, the chin-brow vertical angle (CBVA) is a means to quantify horizontal gaze. While it can be estimated based on visual inspection of a patient standing with hips and knees extended, the CBVA can be more precisely measured on either a clinical photograph or a standing radiograph (Fig. 1). CBVA is the angle subtended by a line drawn between the tip of a patient's chin and the tip of the brow, a line representing the vertical plane, with positive angles indicating downward gaze [26]. Normal CBVA values in an asymptomatic population have not been defined, but postoperative values of approximately $-10^{\circ}$ to $+10^{\circ}$ have been associated with acceptable clinical outcomes [26-28].

After assessing overall alignment, the cervical spine may be more closely examined for the presence of coronal, sagittal and/or rotational deformity. A detailed assessment of neck tenderness, stiffness, and pain with motion 
is critical. Pain and reproduction of neurologic symptoms with neck extension may indicate cervical spinal stenosis or nerve root compression [14]. A decrease in neck and/ or shoulder pain while lying supine is typical in patients with cervical kyphosis as this removes the effect of gravity on their deformity and resultant muscular fatigue that causes pain.

Lastly, a complete neurologic exam should be performed to detect motor weakness, abnormal reflexes, and sensory changes. Signs of myelopathy include difficulty heel-to-toe walking, Romberg's sign, wasting of the intrinsic hand musculature, the finger escape sign, dysdiadochokinesia, diminished sensation, hyperreflexia, clonus, Hoffmann's reflex, the Babinski sign, and the inverted

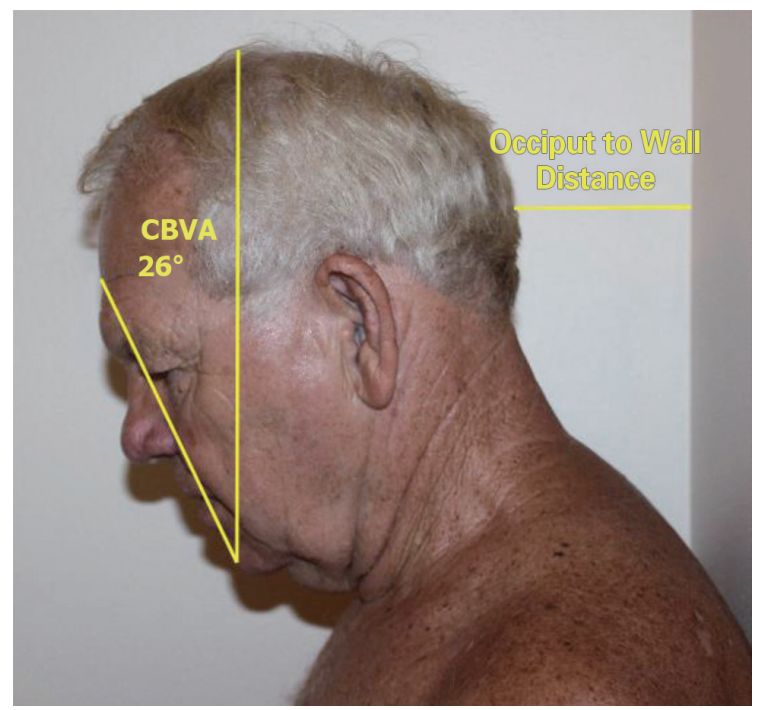

Fig. 1. Clinical photograph showing the measurement of chinbrow vertical angle (CBVA) and occiput to wall distance in a patient with cervical kyphosis. radial reflex. Shoulder girdle muscle wasting may also be observed in patients with C4-C6 stenosis as a result of loss of function of anterior-horn cells in the spinal cord at those levels [14].

\section{Radiographic Evaluation}

Radiographic evaluation of patients with cervical deformities should begin with standing 3-foot anteroposterior and lateral radiographs of the entire spine from the external auditory meatus proximally (estimates the center of mass of the skull) to the femoral heads distally $[23,29]$. The fulllength radiographs should be evaluated critically to determine the location of the primary deformity and any compensatory deformities. Bending or hyperextension views of the entire spine can be obtained as necessary. Dedicated anteroposterior, lateral, and flexion/extension radiographs of the cervical spine should also be obtained $[6,14]$. These studies may demonstrate pathology such as disk narrowing, osteophytes, canal stenosis, ossification of the posterior longitudinal ligament (OPLL), dynamic instability, as well as coronal and sagittal plane deformity [14].

There are several described techniques for quantifying the sagittal plane curvature of the cervical spine (Fig. 2). The Cobb angle method measures the lordosis from either $\mathrm{C} 1$ or $\mathrm{C} 2$ to $\mathrm{C} 7$, using the angle subtended by a line either from the midpoints of the $\mathrm{C} 1$ anterior and posterior tubercles or along the inferior endplate of $\mathrm{C} 2$, to a line drawn along the inferior endplate of C7 [30]. Lordosis is typically indicated by a negative number. The mean $\mathrm{C} 1-$ C7 lordosis is $-41.8^{\circ}$, and the mean C2-C7 lordosis is $-9.6^{\circ}$. Younger patients typically have less lordosis, while
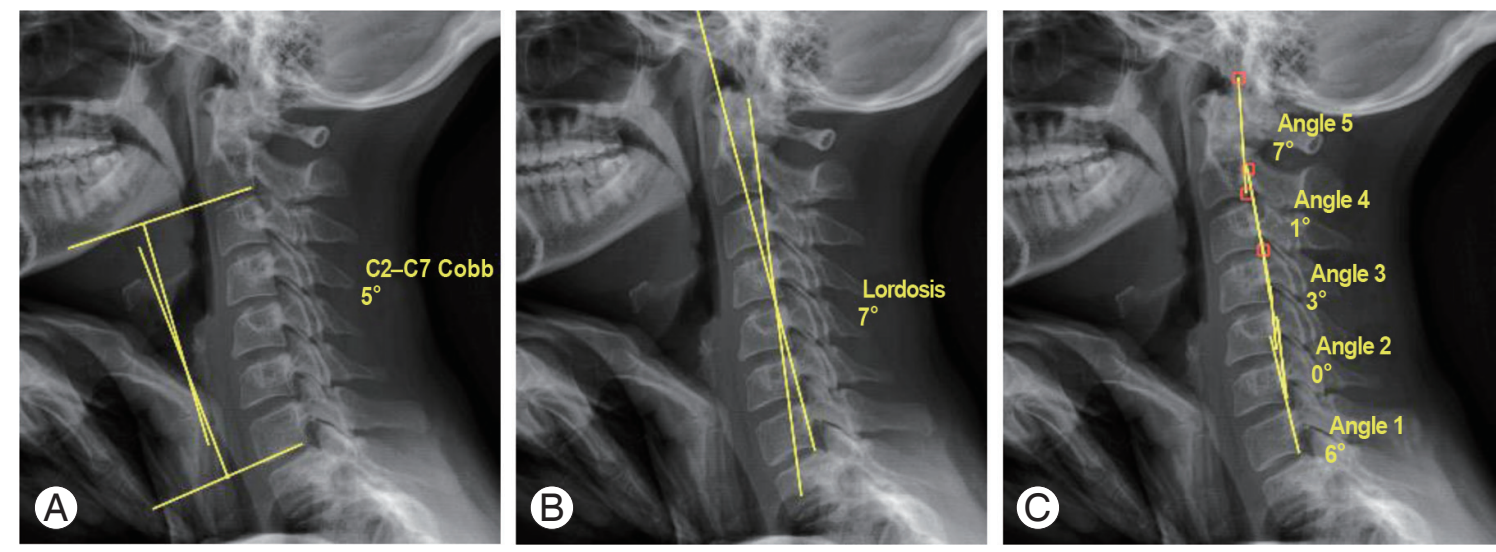

Fig. 2. Lateral EOS images of the cervical spine showing the measurement of cervical lordosis using the Cobb method (A), Jackson physiological stress line method (B), and the Harrison posterior tangent method (C). 
older patients have more as they compensate for a progressive forward shift in the thoracolumbar spine [24,31].

In the Jackson physiological stress line method of measuring cervical sagittal alignment, lines are drawn parallel to the posterior surface of the $\mathrm{C} 2$ and $\mathrm{C} 7$ vertebral bodies, and the angle subtended between them is measured $[32,33]$. The Harrison posterior tangent method is similar to the Jackson method but involves projecting lines parallel to each vertebral body from C2 through C7 and then summing the segmental angles to obtain an overall measure of cervical lordosis or kyphosis [30].

In a direct comparison of the Cobb and Harrison pos-

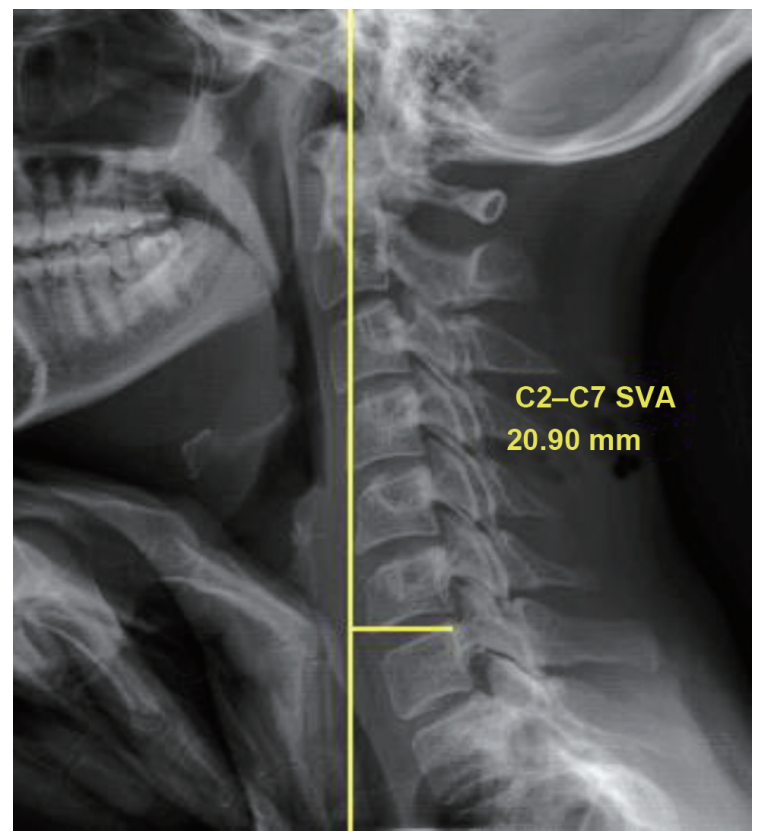

Fig. 3. Lateral EOS image of the cervical spine showing the measurement of C2-C7 sagittal vertical axis (C2-C7 SVA). terior tangent methods, investigators found that the C1$\mathrm{C} 7 \mathrm{Cobb}$ angle tended to overestimate cervical curvature, while the $\mathrm{C} 2-\mathrm{C} 7 \mathrm{Cobb}$ angle underestimated it, and they suggested that the Harrison method was a more reliable technique for quantifying cervical curvature [30]. However, the Cobb method remains the most commonly used because of its ease of use and good inter- and intraexaminer reliability [34].

A complete description of the sagittal profile of the cervical spine depends on both an angular measure as well as a translational measure of the position of the upper cervical spine with respect to $\mathrm{C} 7$. The cervical sagittal vertical axis (SVA) is such a measure of spinal translation, and is necessary to more completely define the deformity. C2C7 SVA is defined as the horizontal distance between the posterior superior aspect of the $\mathrm{C} 7$ vertebral body and a plumb line dropped from the center of the $\mathrm{C} 2$ vertebral body (Fig. 3). Positive values indicate that the center of $\mathrm{C} 2$ body lies anterior to the posterior superior aspect of the C7 body. The normal cervical SVA in asymptomatic individuals has been quantified at $15.6 \pm 11.2 \mathrm{~mm}$, and increasing values (especially those $\geq 40 \mathrm{~mm}$ ) have been shown to negatively impact HRQoL in patients who have undergone posterior cervical fusion surgery $[24,35]$. While lordosis may affect cervical SVA, note that patients with identical cervical Cobb angles may have large differences in their C2-C7 SVA depending on alignment at the cervicothoracic junction.

More recent efforts to evaluate cervical spinal alignment have focused on the interaction between the cervicothoracic junction and the sagittal balance of the cervical spine and head (Fig. 4). Lee et al. [36] defined three
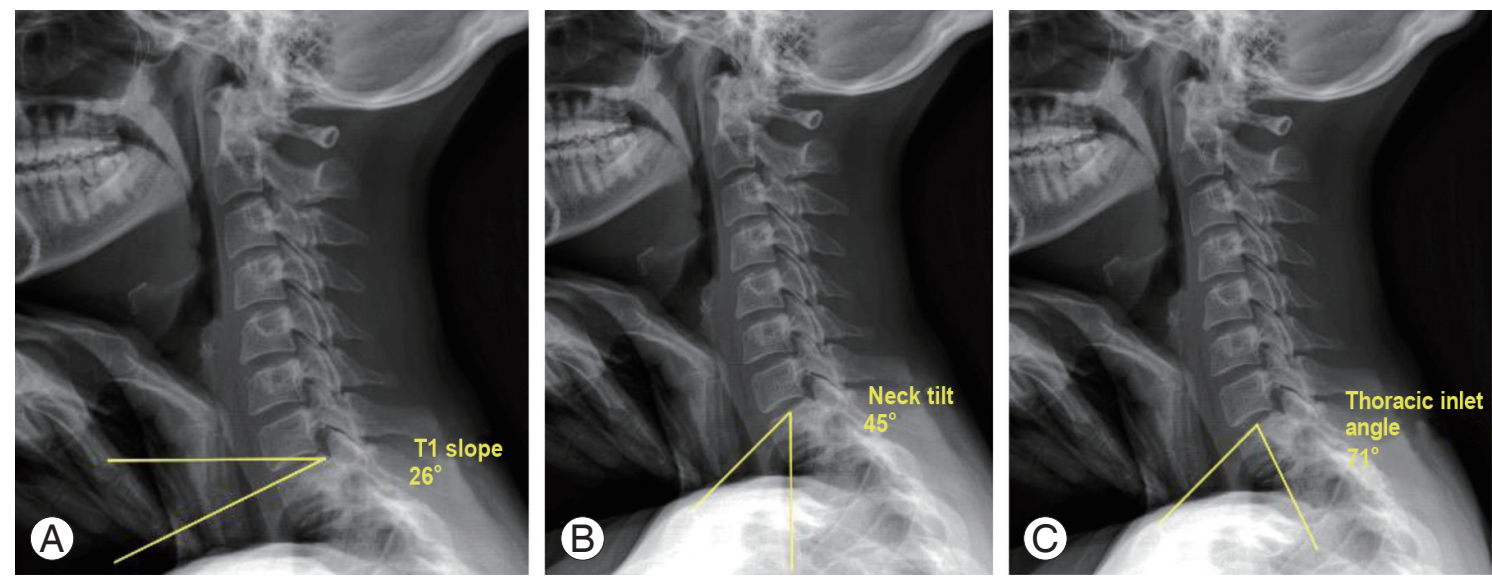

Fig. 4. Lateral EOS images of the cervical spine showing the measurement of the cervicothoracic junction parameters $T 1$ slope $(A)$, neck tilt (B), and thoracic inlet angle or TIA (C). The mathematical relationship between these parameters is TIA=T1 slope+neck tilt. 
relevant parameters: (1) the thoracic inlet angle (TIA) is the angle subtended by a line extending perpendicularly from the center of the T1 upper endplate and a line from the center of the T1 upper endplate to the cephalad aspect of the sternum; (2) the T1 slope (T1S) is the angle between the T1 upper endplate and the horizontal plane; (3) the neck tilt (NT) is the angle between a line extending from the upper end of the sternum to the center of the T1 upper endplate and the vertical plane [36]. Like pelvic incidence, the TIA is a morphometric parameter, which is uninfluenced by posture. The mathematical relationship between these parameters is described by: TIA=T1S+NT.

In addition to plain radiography, magnetic resonance imaging (MRI) is indicated for patients with cervical deformity and long-standing arm or neck pain, neurologic abnormalities, or worsening symptoms. This imaging modality is useful in evaluating soft tissue pathology, such as intervertebral disc herniation and ligamentum flavum hypertrophy and buckling. Furthermore, it can be used to quantify the extent of cord compression, flattening, myelomalacia, and syrinx formation, as well as concomitant

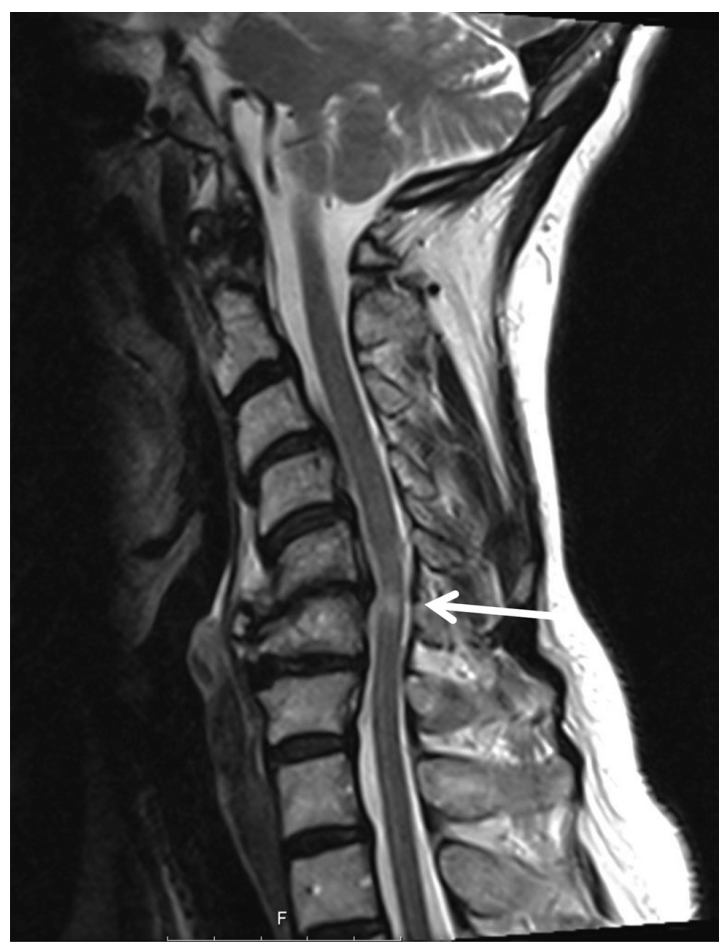

Fig. 5. Mid-sagittal fluid-sensitive magnetic resonance imaging sequence showing severe spinal cord compression and myelomalacia (white arrow) from cervical kyphosis with a central disc herniation and posterior longitudinal ligament hypertrophy. nerve root impingement, if present (Fig. 5). Cord atrophy may be observed in long-standing cases $[6,14]$. These cord changes increase the risk of postoperative neurologic deficit after surgery [6].

Finally, given its superior osseous resolution, computed tomography (CT) may also be considered for preoperative planning purposes. To minimize the risk of intraoperative damage, especially when osteotomies may be needed for correction, the location and course of the vertebral artery should be closely scrutinized using CT angiography or magnetic resonance angiography prior to any cervical deformity surgery [6].

\section{Assessment and Preoperative Planning}

A full assessment of the patient's deformity and neurological status requires careful critique of the history, physical exam, and imaging studies. There is a very limited role for conservative treatment in patients with progressive myelopathy, and these patients should have neural decompression. Concomitantly, these patients should also have correction of their cervical deformity as kyphosis and increased C2-C7 SVA have both been shown to affect spinal cord pressure [9] and neurological outcome in patients undergoing surgery for myelopathy [35,37-40]. In the absence of myelopathy, the indication to correct cervical deformity is in a patient who has failed conservative treatment for loss of horizontal gaze, difficulty swallowing, and/or pain. It is important that the patient has exhausted all potential forms of nonoperative treatment as the surgical procedures necessary to correct cervical deformity are complex and carry significant risk for morbidity.

Currently, no published classification system exists that encompasses all of these factors and guides treatment for patients with cervical deformity. However, there are several key factors which play an important role in the assessment of patients with cervical deformity and affect the choice of operation that is best suited to the patient's particular problem.

\section{Location of deformity}

First, one must determine the primary location of the patient's deformity, which is not always trivial. Because of the close relationship of both global and regional spinal sagittal alignment to the alignment of the cervical spine, it is important to assess the alignment of the spinal column 
in its entirety with full-length standing 36 " radiographs. For example, positive sagittal balance from a primary thoracolumbar deformity leads to hyperlordosis in the cervical spine [31] and conversely, thoracic hypokyphosis in patients with adolescent idiopathic scoliosis leads to a compensatory cervical kyphosis (Fig. 6) [41]. In a similar fashion, deformities at the craniocervical junction or in the upper cervical spine can cause secondary deformities in the subaxial spine and vice versa. Further, the presence of deformities elsewhere in the spine need to be accounted for when planning the deformity correction to maintain overall balance and function. For example, in a patient with subaxial cervical spine kyphosis, fusion of the occipitocervical junction alone in an "optimal" neutral position may leave them with difficulty maintaining horizontal gaze with a markedly positive chin brow vertical angle. Thus, it is important to evaluate the entire spinal axis as well as the lower extremities for all primary and compensatory deformities when planning a surgical correction. When possible, full length skull to foot radiographs should be utilized prior to planning corrective osteotomies for cervical deformity.

\section{Previous surgery}

Many, if not most, patients presenting with cervical deformity will have had previous cervical procedures. The details of what operation was performed, the approach
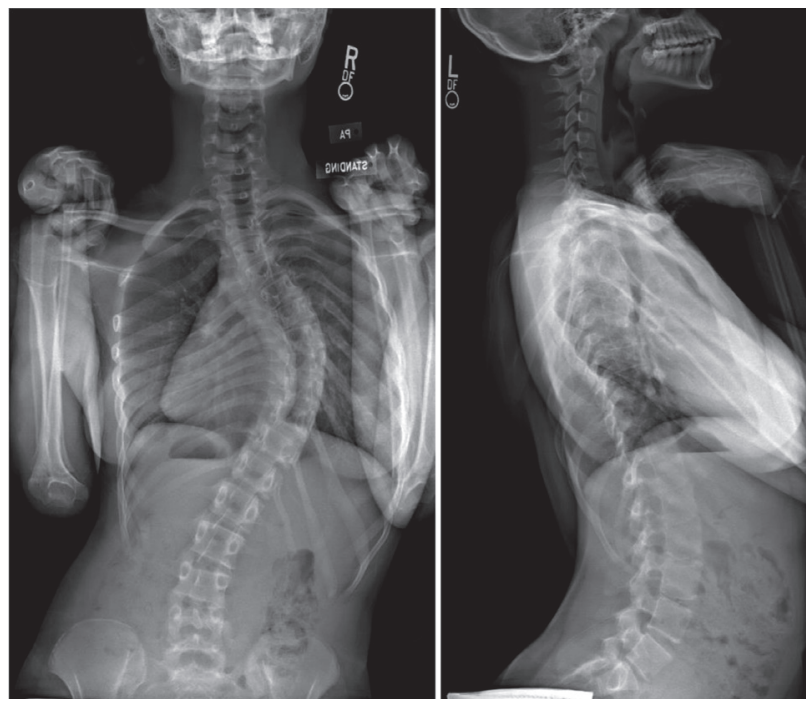

Fig. 6. Lateral scoliosis X-ray of a patient with adolescent idiopathic scoliosis showing a primary thoracic deformity with hypokyphosis and scoliosis resulting in a secondary cervical kyphosis. used, and the success of that operation with respect to decompression and fusion, and whether there were any perioperative or postoperative complications are critical when creating a preoperative plan for revision surgery. If patients have had a previous Smith-Robinson approach, an evaluation by an otolaryngologist is important to determine the innervation status of the recurrent laryngeal nerve (RLN). If a previous operation resulted in an injury to the RLN, then any future anterior approaches should be performed ipsilateral to the injured nerve to avoid the potential for bilateral RLN injuries resulting in devastating vocal cord dysfunction. However, if both RLNs are intact, and an anterior approach is indicated, we recommend an approach from the contralateral side to the previous surgery to avoid dissecting through scar; in essence, turning a revision procedure into a primary procedure to reduce the risk of operative complications.

Additionally, if a patient has had a previous operation, it is important to consider infection or pseudarthrosis, especially with new onset pain after a pain-free interval or in the setting of a newly worsening deformity. If infection is suspected clinically an erythrocyte sedimentation rate (ESR) and C-reactive protein (CRP) should be obtained with a further workup pursued if these markers are elevated. Thin-slice CT scans with coronal and sagittal reconstructions and/or flexion-extension radiographs can be helpful to evaluate for pseudarthrosis [42].

\section{Curve rigidity and degree of deformity}

The rigidity of the curve is an important factor to assess when formulating a preoperative plan for correction of a cervical deformity. If the curve is flexible, then gaining the necessary amount of correction is trivial as the head can be positioned intraoperatively in the desired position and then fused using an anterior, posterior, or a combined approach depending on the amount of immediate stability needed. If the curve is rigid but not ankylosed, then anterior releases through discectomies followed by anterior $+/$ - posterior fusion is often sufficient for achieving the desired result. If however the curve is rigid and ankylosed, then some type of osteotomy will be required to obtain the necessary curve correction. In the setting of a previous operation, a careful review of preoperative imaging studies will allow the surgeon to determine where the spine has been previously fused (anterior or posterior). In general, the spine will need to be approached from the 
side where a previous fusion has been performed (e.g., posterior approach with posterior-based osteotomy in the setting of a prior posterior fusion) to mobilize the spine followed by either same-sided fusion alone, or a combined approach.

The amount of angular and translational deformity in rigid deformities will also affect the surgical plan. Different types of osteotomies can achieve differing amounts of correction (see section on Osteotomy types). A thorough analysis of sites of previous fusion as well as the amount of correction needed will allow the surgeon to formulate an appropriate plan for location and type of osteotomy to be performed. It is important to remember that in patients with a large C2-C7 SVA, osteotomies performed lower in the cervical spine or even upper thoracic spine will allow for the largest amount of translational correction at the time of surgery (Fig. 7).

\section{Neurological status}

Lastly, the presence of myelopathy or radiculopathy in a patient with cervical deformity is important for at least two reasons. First, decompression of the neural elements becomes an important goal of the procedure which could have implications for the bony surfaces available for fusion after deformity correction, or may necessitate both an anterior and posterior fusion due to the iatrogenic instability caused by the decompression. Second, there may be a higher risk of neurological complications after deformity correction in patients who already have a "sick cord" [43].

\section{Osteotomy Types}

Many types of osteotomies have been described in the cervical spine, which are largely similar to ones performed more frequently in the thoracolumbar spine. However, osteotomies in the cervical spine are technically more challenging to perform because of the need to protect the vertebral artery and the potentially devastating consequences of a cervical level cord injury. The various osteotomies can be categorized by approach (anterior vs. posterior vs. combined), or by the number of columns involved in the osteotomy (posterior column osteotomy vs. pedicle subtraction osteotomy or vertebral column resection). Ames and colleagues recently developed a standard nomenclature regarding cervical spine osteotomies that is anatomically-based and graduated in terms of degree of
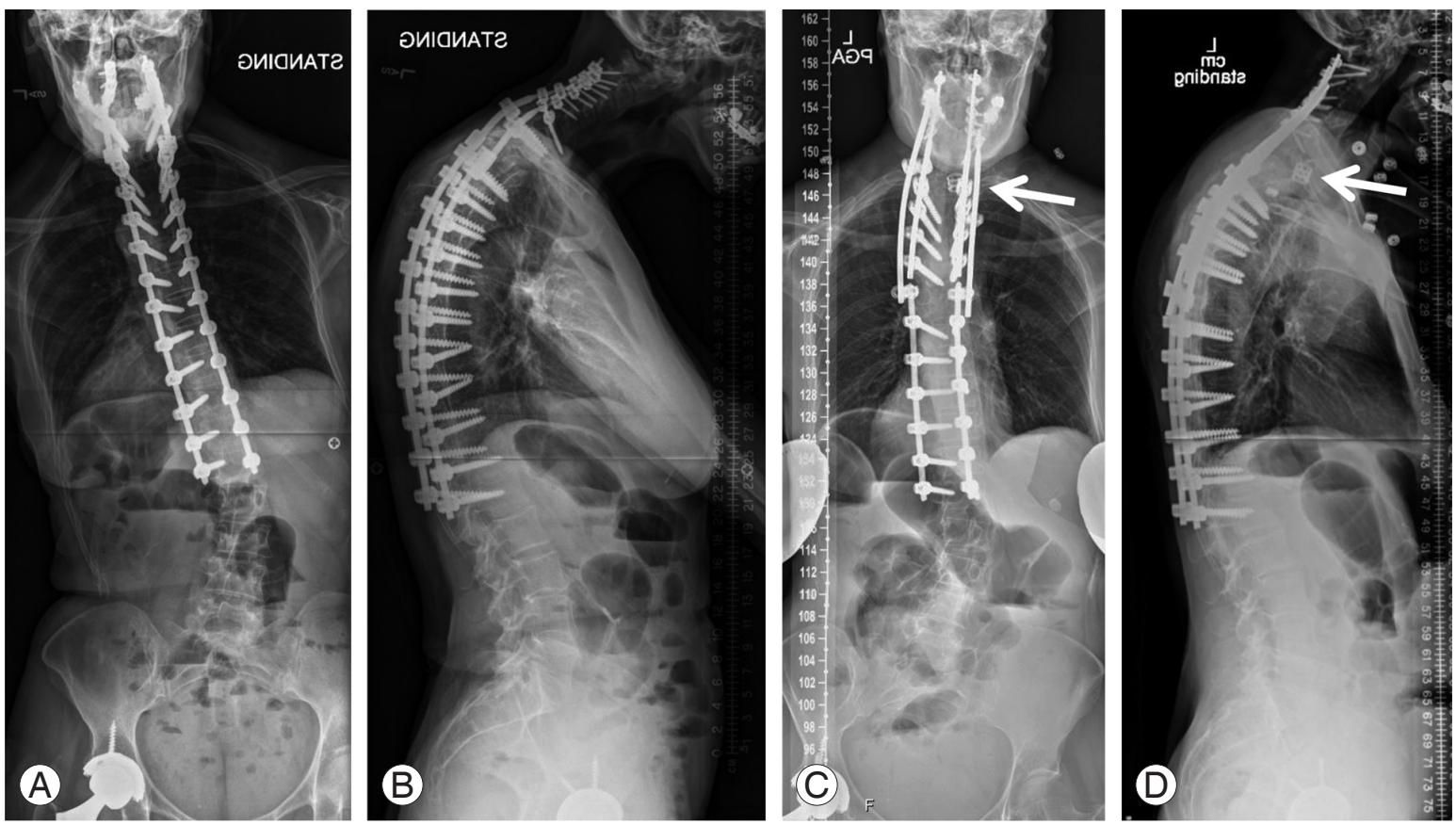

Fig. 7. Preoperative $(\mathbf{A}, \mathbf{B})$ and postoperative $(\mathbf{C}, \mathbf{D})$ lateral full-length spine $X$-rays showing the power of an osteotomy performed at the cervicothoracic junction. In this case, the patient had been previously fused with markedly positive cervical sagittal balance with a large C2-C7 sagittal vertical axis (SVA). A T1 vertebral column resection was performed (arrow) allowing for a large sagittal correction and good restoration of appropriate sagittal balance. 
resection that provides a useful framework to understand the different types of osteotomies [22]. Here we describe the various osteotomies in an anatomically based fashion while highlighting the various degrees of destabilization with each osteotomy.

\section{Anterior osteotomies}

Anterior-based osteotomies can range from partial uncovertebral joint resection as performed during an anterior cervical discectomy and fusion (ACDF), partial or complete corpectomy, or an osteotomy which exits through the uncovertebral joints out laterally into the transverse foramina. These are classified by Ames as grade 1, 3, or 4 osteotomies [22]. These osteotomies depend on motion through the facets to gain correction, and therefore cannot be performed in isolation in patients with fused posterior elements.

The technique for an anterior osteotomy (grade 4) has been described in detail recently by Kim et al. [44]. Briefly, the patient is positioned supine with support under the head in the case of a fixed, kyphotic deformity. Gardner-Wells tongs are placed with $2.2 \mathrm{~kg}$ of traction to stabilize the head. A Smith-Robinson approach is used to approach the convexity of the deformity if there is a concomitant scoliosis in addition to kyphosis. Blunt dissection lateral to the uncinates is performed with a Penfield 4 and/or 2 and is used to identify the fused disc space, which is located just medial to the cranial border of the costal process. Caspar pins are placed in a divergent fashion (perpendicular to the anterior plane of the deformed cervical spine) which can help produce lordosis after completion of the osteotomy. The osteotomy is initiated using a $3 \mathrm{~mm}$ matchstick burr, with the carpentry being tailored to match the patient's unique deformity so that there is proper alignment when the osteotomy is closed down. The bony resection is performed all the way back to the posterior longitudinal ligament. When burring laterally it is imperative to protect the vertebral arteries using a Penfield 4 or 2 , or a cottonoid patty. It is advisable to also perform a foraminotomy at the level of the osteotomy as well to prevent nerve root compression with closure of the osteotomy. With the osteotomy complete, the deformity reduction is performed by removing the supports under the head and pushing on the forehead through the sterile drapes. This can be done in conjunction with distraction along the Caspar pins or by using a laminar spreader in the osteotomy defect. The traction weight is increased to 9 to $11.3 \mathrm{~kg}$ to maintain the correction. A graft with large surface area is shaped to fit the defect to reduce the risk of subsidence, and an anterior cervical plate is placed with fixed angle screws. Adjunctive posterior fusion is performed as necessary for increased stability.

Kim et al. [44] demonstrated that anterior osteotomies provide on average $23^{\circ}$ of angular correction and $1.3 \mathrm{~cm}$ of translational correction. If performed in combination with posteriorly-based Smith-Petersen osteotomies (SPO), they obtained an average angular correction of $33^{\circ}$ and translational correction of $3.7 \mathrm{~cm}$ although most of these were performed only for additional stabilization.

\section{Posterior osteotomies}

Posterior-based osteotomies can range from partial facet joint resection, total facet resection (SPO or Ponte-type), or a closing-wedge pedicle subtraction osteotomy (PSO) involving both the posterior and anterior columns. These have been classified by Ames as grades 1, 2, 5 or 6 depending on the degree of instability produced [22].

The SPO or Ponte-type osteotomy is performed by completely resecting both the superior and inferior articulating facets at a given spinal segment. This may be performed in conjunction with resecting the ligamentum flavum, lamina, and spinous processes. These osteotomies depend on residual anterior column mobility to obtain the necessary correction. These osteotomies are typically performed at multiple levels to obtain increased correction (Fig. 8). Importantly, these osteotomies do not involve any resection of the pedicles or vertebral body. The foramen transversarium is left intact as the vertebral artery runs just anterior to the level of the resection anterior to the facets and exiting nerve roots.

An opening wedge osteotomy, or grade 5 resection, involves complete resection of the posterior elements, lamina, spinous process, and facets followed by osteoclasis with subsequent lengthening of the anterior column. This was originally described by Mason et al. [45] and modified by several people including Urist [46] and McMaster [47], and typically used in the treatment of patients with ankylosing spondylitis. Because it lengthens the anterior column, it is a relatively dangerous osteotomy with significant risks to the anterior soft tissue structures (trachea, esophagus) and is rarely performed. 

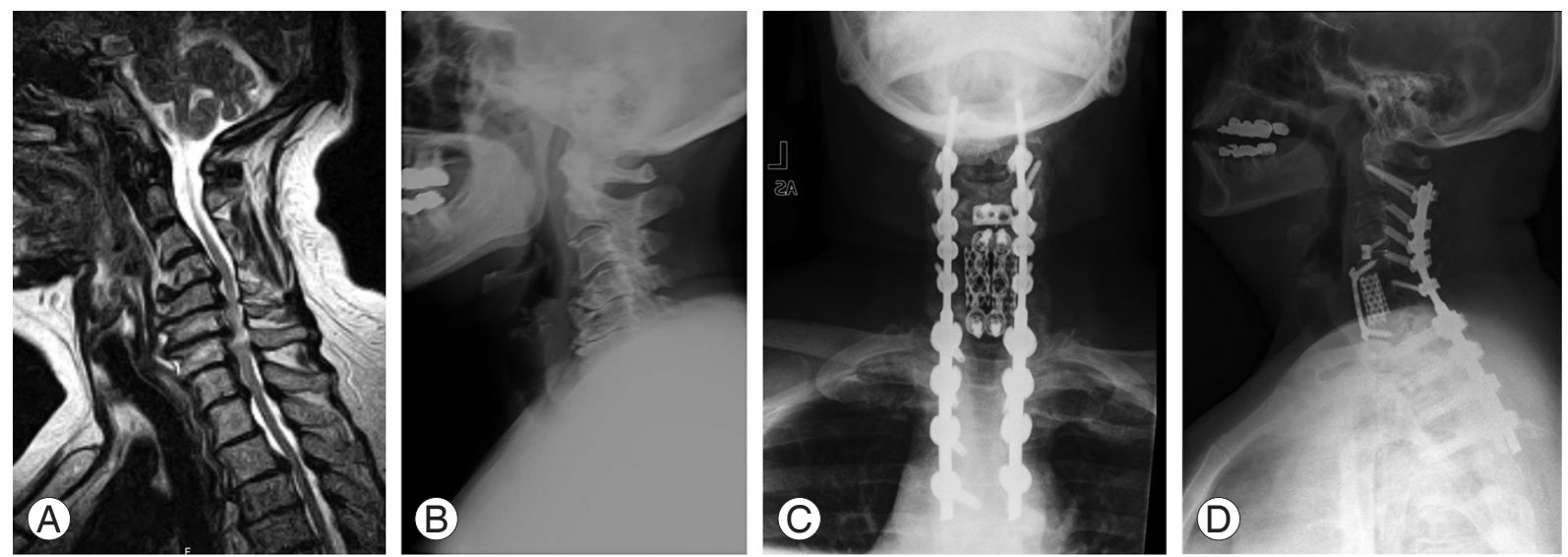

Fig. 8. Preoperative mid-sagittal fluid-sensitive magnetic resonance imaging (A) and lateral (B) radiographs of a patient with cervical kyphosis and multilevel cervical spondylotic myelopathy. The patient underwent an anterior/posterior procedure with an anterior corpectomy, adjacent standalone anterior cervical discectomy and fusion, and posterior fusion with instrumentation crossing the cervicothoracic junction with several Smith-Petersen osteotomies to aid in deformity correction (C, D).

Alternatively, a pedicle subtraction osteotomy (PSO, grade 6) is a spinal shortening closing wedge osteotomy which is inherently safer than the opening wedge osteotomy, in addition to being more stable upon osteotomy closure. Both of these resections are typically performed at the cervicothoracic junction at $\mathrm{C} 7$ for several reasons: (1) the safe location of the vertebral artery in front of the transverse processes of $\mathrm{C} 7,(2)$ the size of the spinal canal at $\mathrm{C} 7-\mathrm{T} 1,(3)$ the long lever arm provided by performing an osteotomy low in the cervical spine which allows for greater translational correction, (4) the mobility of the spinal cord and eighth cervical nerves in this region, and (5) the limited impairment in hand function if a C8 nerve root injury were to occur. Prior to performing a PSO at $\mathrm{C} 7$, however, it is critical to evaluate the preoperative imaging studies carefully to make sure the vertebral artery does not take an aberrant course through the $\mathrm{C} 7$ foramen transversarium, which occurs in $2 \%-5 \%$ of patients [48].

The technique for cervical PSO has been previously described in detail by Wollowick et al. [49]. Briefly, the patient is positioned prone on a Jackson table on bolsters positioned to accommodate the deformity, with the table in maximum reverse Trendelenburg. The head is suspended in Gardner-Wells tongs with $7 \mathrm{~kg}$ traction, with a second traction rope positioned to provide an extension moment after completion of the osteotomy to hold the reduction. Posterior cervical and upper thoracic exposure is performed per routine. If motion is preserved at the craniocervical junction the fusion will stop proximally at $\mathrm{C} 2$, but is taken to the occiput in a completely ankylosed spine for improved fixation. Distal fixation is to T3 or T4. Instrumentation is placed segmentally with lateral mass screws and pedicles screws where appropriate skipping C6 or T1. The osteotomy is begun with a complete laminectomy of $\mathrm{C} 7$ as well as a partial laminectomy of the inferior half of $\mathrm{C} 6$ and superior half of T1. The lateral masses of $\mathrm{C} 7$ are then removed using a rongeur and a high-speed burr. Great care must be taken to remove any overhanging bone on osteotomy closure from the facets of C6 and T1 to prevent $\mathrm{C} 8$ nerve root impingement. After identifying and protecting the thecal sac medially, the $\mathrm{C} 7$ nerve root superiorly, and the $\mathrm{C} 8$ nerve root inferiorly, a burr is used to decancellate the $\mathrm{C} 7$ pedicle and vertebral body. Pituitary rongeurs and reverse-angle curettes are used to remove the bony walls of the pedicle, along with further decancellation of the body. Finally, the dorsal cortex of the vertebral body is impacted into the previously created decancellation cavity to complete the osteotomy. The osteotomy is closed and the deformity corrected by fixing pre-bent rods to the thoracic pedicle screws, and then extending the neck by pulling up on the Gardner-Wells tongs and seating the rods into the cervical lateral mass and/or pedicle screws. The $\mathrm{C} 7$ and $\mathrm{C} 8$ nerve roots again must be meticulously checked to rule out any signs of impingement. The neuromonitoring should also be checked carefully to ensure no signs of spinal cord compromise. Once the deformity is corrected, adjunctive anterior plating with screws above and below the osteotomy can be performed for additional stability.

Kim et al. [50] showed that with isolated cervical PSOs, 
the mean angular correction achieved was $34.5^{\circ}$ with a mean translational correction of $2.5 \mathrm{~cm}$. Interestingly, they found that with combining an anterior osteotomy with posterior SPOs, they achieved a mean angular correction of $27.8^{\circ}$ with a mean translational correction of $2.6 \mathrm{~cm}$, which were both statistically equivalent to those achieved with an isolated PSO, and with significantly less blood loss. Because of this, if technically feasible, they recommend combining anterior osteotomies with SPOs rather than an isolated PSO.

\section{Combined osteotomies}

For severe deformities, both anterior and posterior osteotomies can be combined to achieve significant curve correction. As stated above, it's been shown recently that combining an anterior osteotomy with posterior SPOs can provide similar correction to an isolated PSO [50]. In certain, rare, situations a severe deformity may require removal of a complete vertebral segment (grade 7), consisting of the entire vertebral body, the adjacent disks, the complete uncovertebral joint, lamina, and facets. This type of osteotomy, called a vertebral column resection (VCR), is rarely performed in the cervical spine except for oncologic resections due to the need to protect the vertebral arteries. If necessary, it is performed via a combined approach.

\section{Conclusions}

There remain many unanswered questions regarding the natural history and surgical treatment of cervical deformities, including the role of spinal osteotomies to correct these deformities. Although our understanding of the pathophysiology of cord injury in cervical deformity has increased tremendously in recent years, we still do not know how to accurately predict which patients will have rapid progression of their deformity and/or myelopathy and need earlier, and more aggressive treatment. Our understanding of global spinal sagittal balance is still in its infancy, and future research directed at improving our understanding of TIA and T1S and how that affects cervical spine alignment and positioning of the skull in space will undoubtedly be critical. Importantly, deformity correction to "normal" spinal sagittal alignment may not be appropriate for everyone depending on where the patient lies on the age spectrum and any co-existing spinal or ex- tremity deformities.

The treatment of cervical deformity is complex, but the principles are straightforward - obtaining a balanced correction that results in a solid fusion with adequate neural decompression by performing an operation with the least amount of surgical risk. In cases of a rigid ankylosed cervical spine, osteotomies are necessary to obtain the desired correction which necessarily carry with them increased risk. However, with careful preoperative planning and a full understanding of the anatomic limitations of the various approaches and techniques, one can treat a variety of complex deformities effectively and safely.

\section{Conflict of Interest}

No potential conflict of interest relevant to this article was reported.

\section{References}

1. Cha TD, An HS. Cervical spine manifestations in patients with inflammatory arthritides. Nat Rev Rheumatol 2013;9:423-32.

2. Kubiak EN, Moskovich R, Errico TJ, Di Cesare PE. Orthopaedic management of ankylosing spondylitis. J Am Acad Orthop Surg 2005;13:267-78.

3. Nguyen HV, Ludwig SC, Silber J, et al. Rheumatoid arthritis of the cervical spine. Spine J 2004;4:329-34.

4. O’Shaughnessy BA, Liu JC, Hsieh PC, Koski TR, Ganju A, Ondra SL. Surgical treatment of fixed cervical kyphosis with myelopathy. Spine (Phila Pa 1976) 2008;33:771-8.

5. Scheer JK, Tang JA, Smith JS, et al. Cervical spine alignment, sagittal deformity, and clinical implications: a review. J Neurosurg Spine 2013;19:141-59.

6. Albert TJ, Vacarro A. Postlaminectomy kyphosis. Spine (Phila Pa 1976) 1998;23:2738-45.

7. Breig A, el-Nadi AF. Biomechanics of the cervical spinal cord: relief of contact pressure on and overstretching of the spinal cord. Acta Radiol Diagn (Stockh) 1966;4:602-24.

8. Shimizu K, Nakamura M, Nishikawa Y, Hijikata S, Chiba K, Toyama Y. Spinal kyphosis causes demyelination and neuronal loss in the spinal cord: a new model of kyphotic deformity using juvenile Japanese small game fowls. Spine (Phila Pa 1976) 2005;30:2388-92. 
9. Chavanne A, Pettigrew DB, Holtz JR, Dollin N, Kuntz Ct. Spinal cord intramedullary pressure in cervical kyphotic deformity: a cadaveric study. Spine (Phila Pa 1976) 2011;36:1619-26.

10. Deutsch H, Haid RW, Rodts GE, Mummaneni PV. Postlaminectomy cervical deformity. Neurosurg Focus 2003;15:E5.

11. Iida H, Tachibana S. Spinal cord intramedullary pressure: direct cord traction test. Neurol Med Chir (Tokyo) 1995;35:75-7.

12. Jarzem PF, Quance DR, Doyle DJ, Begin LR, Kostuik JP. Spinal cord tissue pressure during spinal cord distraction in dogs. Spine (Phila Pa 1976) 1992;17:S22734.

13. Tachibana S, Kitahara Y, Iida H, Yada K. Spinal cord intramedullary pressure. A possible factor in syrinx growth. Spine (Phila Pa 1976) 1994;19:2174-8.

14. Emery SE. Cervical spondylotic myelopathy: diagnosis and treatment. J Am Acad Orthop Surg 2001;9: 376-88.

15. Herman JM, Sonntag VK. Cervical corpectomy and plate fixation for postlaminectomy kyphosis. J Neurosurg 1994;80:963-70.

16. Nagano A, Miyamoto K, Fushimi K, Hosoe H, Shimizu K. Failure of reconstruction surgery using anterior fibular strut grafting to correct postlaminectomy kyphosis. J Clin Neurosci 2007;14:376-9.

17. Park Y, Riew KD, Cho W. The long-term results of anterior surgical reconstruction in patients with postlaminectomy cervical kyphosis. Spine J 2010;10: 380-7.

18. Zdeblick TA, Bohlman HH. Cervical kyphosis and myelopathy: treatment by anterior corpectomy and strut-grafting. J Bone Joint Surg Am 1989;71:170-82.

19. Rhee JM, Shamji MF, Erwin WM, et al. Nonoperative management of cervical myelopathy: a systematic review. Spine (Phila Pa 1976) 2013;38:S55-67.

20. Nolan JP Jr, Sherk HH. Biomechanical evaluation of the extensor musculature of the cervical spine. Spine (Phila Pa 1976) 1988;13:9-11.

21. Ganju A, Ondra SL, Shaffrey CI. Cervical kyphosis. Tech Orthop 2003;17:345-54.

22. Ames CP, Smith JS, Scheer JK, et al. A standardized nomenclature for cervical spine soft-tissue release and osteotomy for deformity correction: clinical article. J Neurosurg Spine 2013;19:269-78.

23. Joseph SA Jr, Moreno AP, Brandoff J, Casden AC, Ku- flik P, Neuwirth MG. Sagittal plane deformity in the adult patient. J Am Acad Orthop Surg 2009;17:37888.

24. Hardacker JW, Shuford RF, Capicotto PN, Pryor PW. Radiographic standing cervical segmental alignment in adult volunteers without neck symptoms. Spine (Phila Pa 1976) 1997;22:1472-80.

25. Bridwell KH, Lenke LG, Lewis SJ. Treatment of spinal stenosis and fixed sagittal imbalance. Clin Orthop Relat Res 2001;(384):35-44.

26. Suk KS, Kim KT, Lee SH, Kim JM. Significance of chin-brow vertical angle in correction of kyphotic deformity of ankylosing spondylitis patients. Spine (Phila Pa 1976) 2003;28:2001-5.

27. Deviren V, Scheer JK, Ames CP. Technique of cervicothoracic junction pedicle subtraction osteotomy for cervical sagittal imbalance: report of 11 cases. J Neurosurg Spine 2011;15:174-81.

28. Zheng GQ, Song K, Zhang YG, et al. Two-level spinal osteotomy for severe thoracolumbar kyphosis in ankylosing spondylitis. Experience with 48 patients. Spine (Phila Pa 1976) 2014;39:1055-8.

29. Ames CP, Blondel B, Scheer JK, et al. Cervical radiographical alignment: comprehensive assessment techniques and potential importance in cervical myelopathy. Spine (Phila Pa 1976) 2013;38:S149-60.

30. Harrison DE, Harrison DD, Cailliet R, Troyanovich SJ, Janik TJ, Holland B. Cobb method or Harrison posterior tangent method: which to choose for lateral cervical radiographic analysis. Spine (Phila Pa 1976) 2000;25:2072-8.

31. Kim HJ, Lenke LG, Oshima Y, et al. Cervical Lordosis Actually Increases With Aging and Progressive Degeneration in Spinal Deformity Patients. Spine Deformity 2014;2:410-4.

32. Gore DR. Roentgenographic findings in the cervical spine in asymptomatic persons: a ten-year follow-up. Spine (Phila Pa 1976) 2001;26:2463-6.

33. Jackson R. The cervical syndrome. 2nd ed. Springfield, IL; 1958.

34. Cote P, Cassidy JD, Yong-Hing K, Sibley J, Loewy J. Apophysial joint degeneration, disc degeneration, and sagittal curve of the cervical spine. Can they be measured reliably on radiographs? Spine (Phila $\mathrm{Pa}$ 1976) 1997;22:859-64.

35. Tang JA, Scheer JK, Smith JS, et al. The impact of standing regional cervical sagittal alignment on out- 
comes in posterior cervical fusion surgery. Neurosurgery 2012;71:662-9.

36. Lee SH, Kim KT, Seo EM, Suk KS, Kwack YH, Son ES. The influence of thoracic inlet alignment on the craniocervical sagittal balance in asymptomatic adults. J Spinal Disord Tech 2012;25:E41-7.

37. Suda K, Abumi K, Ito M, Shono Y, Kaneda K, Fujiya M. Local kyphosis reduces surgical outcomes of expansive open-door laminoplasty for cervical spondylotic myelopathy. Spine (Phila Pa 1976) 2003;28:125862.

38. Baba H, Uchida K, Maezawa Y, Furusawa N, Azuchi M, Imura S. Lordotic alignment and posterior migration of the spinal cord following en bloc open-door laminoplasty for cervical myelopathy: a magnetic resonance imaging study. J Neurol 1996;243:626-32.

39. Ferch RD, Shad A, Cadoux-Hudson TA, Teddy PJ. Anterior correction of cervical kyphotic deformity: effects on myelopathy, neck pain, and sagittal alignment. J Neurosurg 2004;100:13-9.

40. Villavicencio AT, Babuska JM, Ashton A, et al. Prospective, randomized, double-blind clinical study evaluating the correlation of clinical outcomes and cervical sagittal alignment. Neurosurgery 2011;68: 1309-16.

41. Hilibrand AS, Tannenbaum DA, Graziano GP, Loder RT, Hensinger RN. The sagittal alignment of the cervical spine in adolescent idiopathic scoliosis. J Pediatr Orthop 1995;15:627-32.

42. Song KS, Piyaskulkaew C, Chuntarapas T, et al. Dy- namic radiographic criteria for detecting pseudarthrosis following anterior cervical arthrodesis. J Bone Joint Surg Am 2014;96:557-63.

43. Kim HJ, Nemani VM, Daniel Riew K. Cervical osteotomies for neurological deformities. Eur Spine J 2015;24 Suppl 1:16-22.

44. Kim HJ, Piyaskulkaew C, Riew KD. Anterior cervical osteotomy for fixed cervical deformities. Spine (Phila Pa 1976) 2014;39:1751-7.

45. Mason C, Cozen L, Adelstein L. Surgical correction of flexion deformity of the cervical spine. Calif Med 1953;79:244-6.

46. Urist MR. Osteotomy of the cervical spine; report of a case of ankylosing rheumatoid spondylitis. J Bone Joint Surg Am 1958;40:833-43.

47. McMaster MJ. Osteotomy of the cervical spine in ankylosing spondylitis. J Bone Joint Surg Br 1997;79: 197-203.

48. Wakao N, Takeuchi M, Kamiya M, et al. Variance of cervical vertebral artery measured by CT angiography and its influence on C7 pedicle anatomy. Spine (Phila Pa 1976) 2014;39:228-32.

49. Wollowick AL, Kelly MP, Riew KD. Pedicle subtraction osteotomy in the cervical spine. Spine (Phila Pa 1976) 2012;37:E342-8.

50. Kim HJ, Piyaskulkaew C, Riew KD. Comparison of smith-petersen osteotomy versus pedicle subtraction osteotomy versus anterior-posterior osteotomy types for the correction of cervical spine deformities. Spine (Phila Pa 1976) 2015;40:143-6. 